\title{
O Uso de Métodos de Avaliação do Estado Nutricional e Cálculo de Necessidade Energética por Nutricionistas em Dois Hospitais Públicos do Rio de Janeiro
}

\section{The Use of Nutritional Status Evaluation and Energy Needs Calculation Methods by Nutritionists in Two Public Hospitals of Rio de Janeiro}

\section{RESUMO}

Introdução: O estado nutricional e a avaliação do gasto metabólico têm impacto significativo na evolução clínica dos pacientes. Objetivo: Identificar quais métodos de avaliação do estado nutricional e o cálculo da necessidade energética têm sido eleitos na prática clínica de nutricionistas, conhecendo os motivos que influenciaram suas escolhas. Metodologia: Aplicou-se um questionário semiestruturado, com perguntas quanti e qualitativas, por meio do autopreenchimento. Resultados: A maioria dos entrevistados era do gênero feminino, formados em instituições públicas, com formação complementar. O principal local de atuação foi a internação e o único equipamento comum a todos foi a balança. Os métodos escolhidos por $100 \%$ dos profissionais foram o IMC e o método $\mathrm{Kcal} / \mathrm{Kg}$ de peso. Conclusão: Apesar do conhecimento teórico-prático de diferentes métodos de avaliação nutricional e do cálculo da necessidade energética houve preferência pelos de maior facilidade e praticidade, havendo pouco reconhecimento de suas limitações. Entre os motivos para tais escolhas destacam-se a falta de equipamentos e o número de profissionais por enfermaria.

DESCRITORES: Equações Preditivas, Avaliação Nutricional, Nutricionista.

\begin{abstract}
Introduction: Nutritional status and the evaluation of metabolic expenditure have a significant impact on the clinical evolution of patients. Objective: To identify which nutritional status and energy need assessment methods have been chosen in the clinical practice of nutritionists, knowing the possible reasons that influenced their choices. Methodology: For the study, a semi-structured questionnaire was developed, with quanti and qualitative questions and the data was collected through self-completion. Results: Most of the interviewees were female, graduated in public institutions and all of them had made complementary studies. The main place of work of the professionals was the hospitalization and the only equipment present common to all nutritionists was the scale. The chosen methods by $100 \%$ were the $\mathrm{BMI}$ and the $\mathrm{Kcal} / \mathrm{Kg}$ body weight method. Conclusion: Despite the theoretical and practical knowledge of different methods of nutritional assessment and calculation of the energy requirement, there was a preference for the easiest and most practical methods, with little recognition of their limitations. Other reasons include the lack of equipment and the number of professionals per ward.
\end{abstract}

DESCRIPTORS: Predictive Equations, Nutrition Assessment, Nutritionist.

1 - Universidade Federal do Estado do Rio de Janeiro - UNIRIO. 
$\mathrm{O}$ estado nutricional tem impacto significativo na evolução clínica dos pacientes ${ }^{1}$, influenciando o tempo de permanência nos hospitais e o custo ${ }^{2}$. Nessa perspectiva, o aporte inadequado de nutrientes pode ocasionar alterações nesse estado nutricional, contribuindo para a elevação dos índices de morbi-mortalidade ${ }^{3}$.

Desnutrição proteico energética, síndrome de realimentação, cetose são algumas possíveis complicações de condutas nutricionais inadequadas $^{2,4,5}$. Entre elas, a desnutrição é o problema mais prevalente em pacientes hospitalizados no Brasil ${ }^{2}$.

A avaliação do gasto metabólico é um dos principais pontos para o êxito da terapia nutricional. A melhora e/ou manutenção do estado nutricional dependem dessa precisão, pois tanto a hipoalimentação quanto a hiperalimentação podem ocasionar efeitos deletérios na condição de saúde ${ }^{6}$.

Existem diversos indicadores para avaliação nutricional - avaliação clínica, bioquímica, antropométrica e de composição corporal e inquéritos alimentares ${ }^{3}$. Porém, nenhum deles quando isolado pode ser considerado padrão ouro, por todos terem vantagens e limitações, sendo necessária a escolha adequada de um ou mais métodos de avaliação de acordo com a população e/ou condições a serem avaliadas ${ }^{7}$.

Da mesma forma, existem vários métodos para determinar o gasto energético, como calorimetria, sensores de calor e movimento, registros de atividade física, equações preditivas ${ }^{8}$, entre outros. A calorimetria e o método da água duplamente marcada são considerados os mais precisos. No entanto, têm um custo elevado, requerendo pessoal treinado, além de demoram mais tempo para serem realizados ${ }^{4}$.

Ao longo dos tempos têm sido desenvolvidas várias equações, numa tentativa de estimar mais facilmente o metabolismo basal ou de repou$\mathrm{so}^{7}$ e algumas delas têm sido mais utilizadas na prática clínica ${ }^{9}$. Todavia, outros métodos de cálculo também têm sido propostos, como é o caso das recomendações de Kcal por Kg de peso. Bottoni et al' classificam tais recomendações como "fórmula de bolso"; um método rápido, porém empírico, que estima o gasto energético apenas a partir do peso e não leva em consideração a gravidade do paciente (quadro clínico e metabólico).

Diversos estudos apontam a possibilidade de erro nos resultados encontrados a partir do uso das equações preditivas, destacando o fato de não haver até os dias atuais propostas com base na população brasileira ${ }^{5,10}$. Em relação ao método $\mathrm{Kcal} / \mathrm{Kg}$ de peso são estabelecidas amplas faixas de recomendações de calorias, de acordo com tipos de pacientes ${ }^{11}$ e o valor escolhido deve ser multiplicado, apenas, pela variável peso. Tanto a escolha do valor a ser multiplicado quanto o peso utilizado influenciarão nos resultados ${ }^{5}$.

Apesar dos possíveis erros, a estimativa de gasto energético, por meio de equações de predição, continua sendo recomendada por ser menos onerosa e de aplicabilidade mais simples ${ }^{12}$, assim como o método $\mathrm{Kcal} / \mathrm{Kg}$ de peso também tem sido amplamente divulgado ${ }^{13,14,15}$.

Dada à relevância que o estado nutricional e as condutas dietoterápicas exercem na condição clínica dos pacientes, este estudo objetivou identificar quais métodos de avaliação do estado nutricional e do cálculo de necessidade energética têm sido eleitos na prática clínica de nutricionistas, em dois hospitais públicos do tipo geral, conhecendo os possíveis motivos que influenciaram suas escolhas.

\section{Metodologia}

O presente estudo compreende dados do Projeto de Pesquisa intitulado "A Escolha de Equações Preditivas na Prática Clínica do Nutricionista", um estudo do tipo transversal, realizado desde 2015 em hospitais federais do Município do Rio de Janeiro.

Considerou-se como critério de inclusão a concordância prévia da Direção da unidade de saúde e o consentimento do profissional nutricionista em participar da pesquisa, através da assinatura do Termo de Consentimento Livre e Esclarecido.

Para a realização do estudo foi elaborado um questionário semiestruturado, com perguntas quanti e qualitativas. Os dados foram coletados por meio do autopreenchimento e a amostra do presente trabalho foi obtida entre junho de 2015 e outubro de 2016.

Procedeu-se a análise exploratória dos dados quantitativos utilizando-se o programa Microsoft Excel, versão 2007, para construção de frequências absolutas e relativas, representadas em tabela e gráficos. 
Para a análise do estudo descritivo, os dados obtidos foram transcritos para tabelas no programa Microsoft Word, com a finalidade de facilitar a leitura das respostas qualitativas, e também com o objetivo de comparar as respostas dos entrevistados. Procurou-se extrair e agrupar as respostas mais comuns em cada pergunta. E para expor melhor os resultados, as falas dos nutricionistas foram utilizadas na discussão do trabalho.

O projeto foi aprovado pelo comitê de pesquisa UNIRIO, conforme Parecer CEP n 468.053 e está registrado no Departamento de Pesquisa da UNIRIO sob n P0012/2015.

\section{Resultados}

Entre junho de 2015 e outubro de 2016, foram convidados a participar do estudo os 6 hospitais públicos federais existentes no Município do Rio de Janeiro, porém, apenas 2 hospitais do tipo geral aceitaram participar completamente da pesquisa, tendo 1 respondido incompletamente à solicitação e o restante não respondido ao contato feito pela Coordenação Geral de Assistência da Secretaria de Atenção à Saúde. Do total de 65 nutricionistas, lotados nas duas unidades participantes, apenas 32 responderam ao questionário. A caracterização dessa amostra encontra-se na Tabela 1.

A maioria dos entrevistados foi do gênero feminino e graduou-se em instituição pública, tendo a maior parte concluído o seu curso há pelo menos 10 anos. Poucos (18,75\%), graduaram-se recentemente (há menos de 5 anos).

Tabela 1 - Caracterização dos nutricionistas participantes

\begin{tabular}{|c|c|c|}
\hline Características & $n=32$ & $\%$ \\
\hline $\begin{array}{l}\text { Sexo } \\
\text { Feminino } \\
\text { Masculino }\end{array}$ & $\begin{array}{l}31 \\
01\end{array}$ & $\begin{array}{c}96,87 \\
3,12\end{array}$ \\
\hline $\begin{array}{l}\text { Instituição de Graduação } \\
\text { Pública } \\
\text { Privada }\end{array}$ & $\begin{array}{l}23 \\
09\end{array}$ & $\begin{array}{l}71,87 \\
28,12\end{array}$ \\
\hline $\begin{array}{l}\text { Tempo de formado } \\
\leq 5 \text { anos } \\
5 \text { anos }-10 \text { anos } \\
\geq 10 \text { anos }\end{array}$ & $\begin{array}{l}06 \\
12 \\
14\end{array}$ & $\begin{array}{l}18,75 \\
37,50 \\
43,75\end{array}$ \\
\hline $\begin{array}{l}\text { Formação Complementar } \\
\text { Sim } \\
\text { Não }\end{array}$ & $\begin{array}{l}32 \\
-\end{array}$ & $\begin{array}{c}100,00 \\
-\end{array}$ \\
\hline $\begin{array}{l}\text { Tipo de Formação Complementar } \\
\text { Pequena duração (atual aperfeiçoamento) } \\
\text { Média duração (especialização) } \\
\text { Longa duração (Mestrado e doutorado) }\end{array}$ & $\begin{array}{l}24 \\
26 \\
07\end{array}$ & $\begin{array}{l}75,00 \\
81,25 \\
21,87\end{array}$ \\
\hline $\begin{array}{l}\text { Tempo da última Formação Complementar } \\
\leq 5 \text { anos } \\
5 \text { anos }-10 \text { anos } \\
\geq 10 \text { anos }\end{array}$ & $\begin{array}{l}24 \\
05 \\
03\end{array}$ & $\begin{array}{l}75,00 \\
15,62 \\
09,37\end{array}$ \\
\hline $\begin{array}{l}\text { Local de atuação } \\
\text { Ambulatório } \\
\text { Internação } \\
\text { Administrativo } \\
\text { Ambulatório e internação } \\
\text { Administrativo e internação }\end{array}$ & $\begin{array}{c}02 \\
25 \\
1 \\
2 \\
2\end{array}$ & $\begin{array}{l}06,25 \\
78,12 \\
03,12 \\
06,25 \\
06,25\end{array}$ \\
\hline
\end{tabular}


Todos os participantes referiram formação complementar, em sua maioria concluídos há menos de 5 anos. A maior parte referiu formação complementar de média duração (especialização), seguidos pelos com formação de pequena duração (atualização e aperfeiçoamento). E, apenas $21,87 \%$, relataram ter cursos de longa duração (mestrado e doutorado).

As principais áreas de formação complementar citadas foram: nutrição do adulto, nutrição funcional, saúde pública e coletiva, e outras, sem especificarem qual(is). $\mathrm{E}$ as menos citadas foram: nutrição em alimentação coletiva, nutrição do idoso e nutrição esportiva. Dentre os entrevistados $40,62 \%$ referiram mais de uma área de formação complementar (Figura 1).

Dos participantes, $81,25 \%$ relataram que a formação complementar contribuiu para sua atuação no local de trabalho. Abaixo foram destacadas algumas falas:

"Sim. Minha atuação profissional tornou-se mais completa e com mais foco".

"Maior eficiência em termos de avaliação nutricional, cálculo de necessidades calóricas e protéicas, etc". "Sim, muitas vezes na forma de agir dentro da instituição".

"Na escuta, humanização".

"Sim, fiz especialização lato e stricto sensu e residência em saúde pública e hoje faço parte do núcleo de planejamento."

"O conhecimento adquirido na formação complementar me ajudou na dietoterapia dos pacientes."

O número de leitos informado variou de 30 a 50 por enfermaria, e o número de nutricionistas foi sempre de 1 profissional por enfermaria.

O principal local de atuação dos profissionais foi a internação, apenas 6,25\% trabalhavam em ambulatórios e 6,25\% atuavam em ambulatórios e internação. Poucos $(3,12 \%)$ referiram ocupar cargos apenas administrativos e 6,25\% relataram atuar nas atividades administrativas associada a internação.

De acordo com a Figura 2 é possível verificar que os profissionais contam como principais recursos materiais para a avaliação do estado nutricional dos pacientes atendidos: balança, estadiômetro e adipômetro. Todos os profissionais que atuavam em ambulatório referiram ter balança e estadiômetro, mas nem todos que atuavam na internação referiram ter estadiômetro. Somente $15,62 \%$ dos profissionais, relataram ter acesso a balança junto ao leito hospitalar e, 9,37\% a bioimpedância, todos da mesma unidade hospitalar.

A prática de avaliar o estado nutricional dos pacientes foi relatada por todos os profissionais, sendo o cálculo do Índice de Massa Corporal - IMC o parâmetro escolhido por $100 \%$ deles. Apenas $9,37 \%$ dos nutricionistas também relataram a

Figura 1. Área de formação complementar dos nutricionistas.

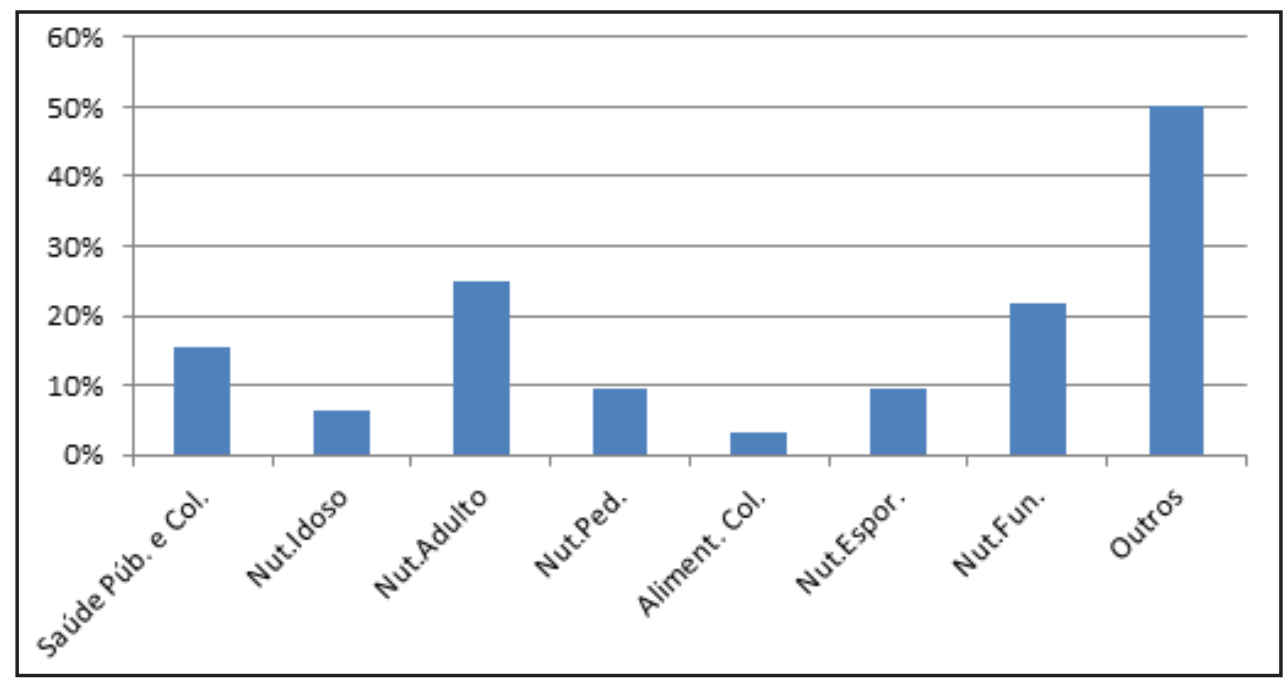


prática de avaliar circunferências, destes, somente um mencionou realizar a avaliação de dobras cutâneas. O uso de outras medidas foi apontado por $31,25 \%$, sem especificação de qual.

Ao responderem sobre o conhecimento de métodos de cálculo do Gasto Energético Total - GET (Figura 3) a maioria dos profissionais relatou conhecer, principalmente, o método Kcal/ Kg de peso; as equações de Harris e Benedict, da Organização das Nações Unidas para Agricultura e Alimentação - FAO / Organização Mundial da Saúde - OMS; e as fórmulas da Ingestão Dietética de Referência - IDR. Enquanto, a Calorimetria indireta - VO2/CO2, as equações de Schofield e o método da Água Duplamente Marcada foram os menos conhecidos. O Frequencímetro foi desconhecido por todos os profissionais entrevistados. Alguns ainda relataram conhecer outro(s) método(s), além dos listados, porém não informaram qual(is).

Poucos profissionais $(18,75 \%)$ referiram não estimar o GET dos pacientes e todos os que disseram calcular apontaram o método Kcal/Kg de peso como o eleito para tal, independentemente, do local de atuação ser internação ou ambulatório. Os motivos para a escolha do método $\mathrm{Kcal} / \mathrm{Kg}$ de peso foram apontados por todos os nutricionistas como praticidade e rapidez, conforme relatos a seguir:

"É prático, objetivo diante do número de pacientes que temos para atender em um curto tempo. Não identifiquei limitação do mesmo."

Figura 2. Equipamentos disponíveis nas unidades hospitalares para o uso do nutricionista.

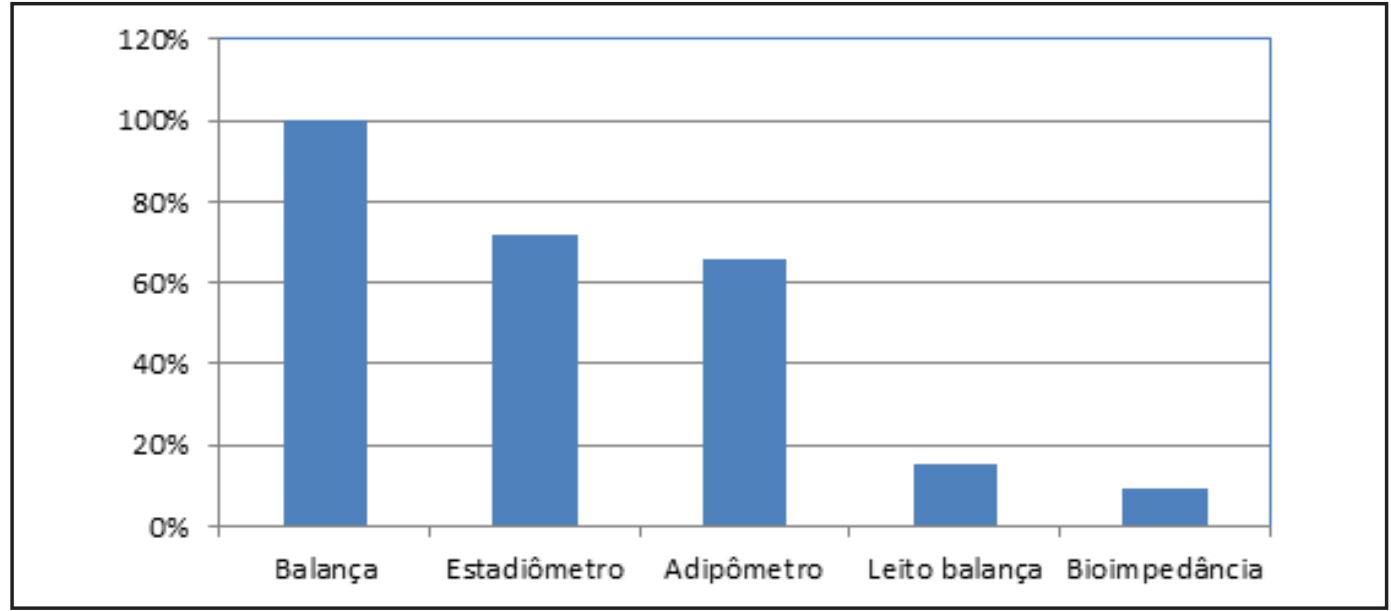

Figura 3. Métodos de cálculo do Gasto Energético Total conhecidos pelos nutricionistas.

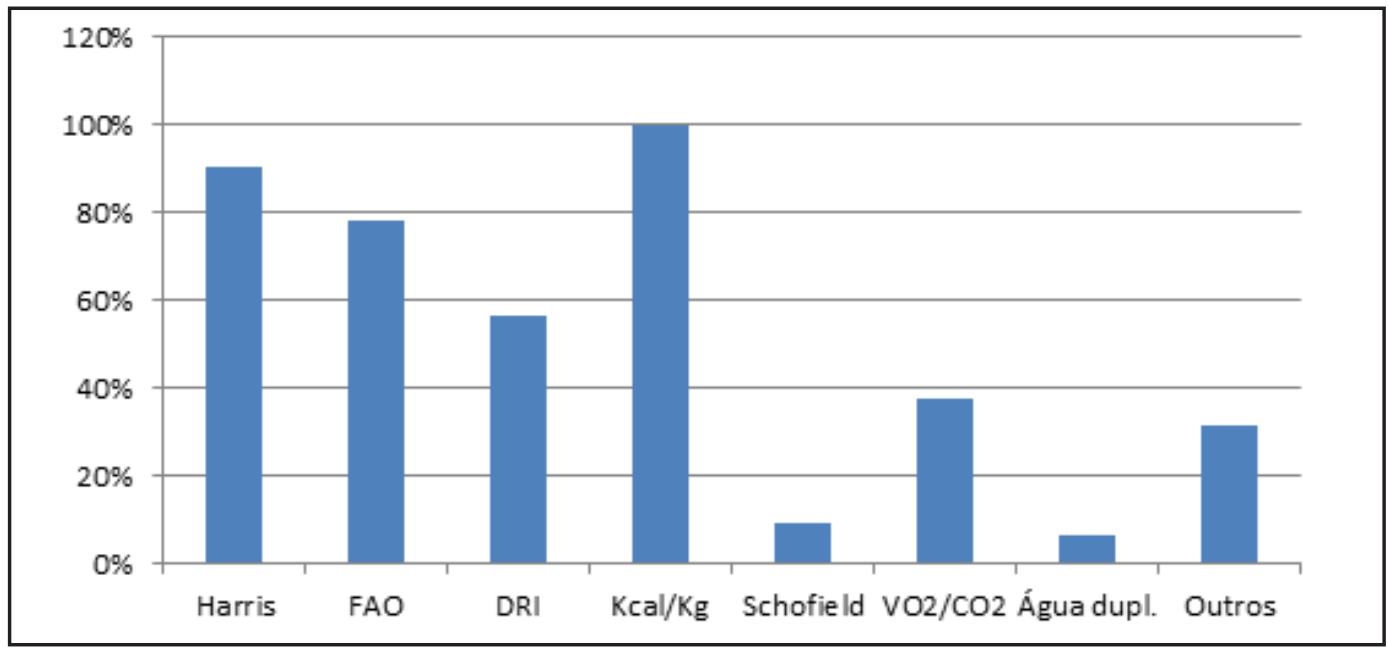


"É um método prático, não tem diferença significativa com outro método." "O método ao que se aplica atende bem. Não uso de fórmulas e medidas mais específicas por não ter tempo disponível de atendimento individual." "É um método prático cujo resultado não difere significativamente de outras equações preditivas "Harris \& Benedict", por exemplo. Não tenho identificado limitação no uso de Kcal/ Kg de peso corpóreo."

"Método prático rapidamente calculado e de fácil uso no dia-a-dia. Bastante utilizado em pesquisas e artigos científicos, tendo respaldo técnico."

"Método prático, que independe de equipamentos complexos, facilmente calculável."

"Método utilizado no serviço."

Alguns entrevistados relataram também usar outras fórmulas para estabelecer o GET, além do método $\mathrm{Kcal} / \mathrm{Kg}$ de peso. Entre os que trabalhavam na área de internação, as equações preditivas citadas foram: Harris e Benedict $(9,37 \%)$, FAO/OMS $(3,12 \%)$ e IDR $(6,25 \%)$. E entre os que atuavam em ambulatório, as fórmulas da $\mathrm{FAO} /$ OMS $(3,12 \%)$.

Somente $12,50 \%$ dos profissionais $(6,25 \%$ atuantes na internação e $6,25 \%$ no ambulatório) mencionaram verificar limitações no referido método, justificando a necessidade do uso de outra forma de cálculo, além do Kcal/Kg de peso, de acordo com os relatos a seguir:

"Pode subestimar necessidade calórica".

"Algumas vezes acredito que gere valores superestimados, por isso faço uma média de resultados de métodos diferentes."

"Na minha opinião é um bom método, mas apresenta limitações, pois não é possível um cálculo preciso das necessidades energéticas do paciente." "Não é a melhor forma para cálculo de gasto energético."

\section{Discussão}

Conforme apontado, a amostra do estudo caracterizou-se, predominantemente, por nutricionistas do gênero feminino, graduadas em instituição pública há pelo menos 10 anos, recentemente pós-graduadas em cursos de pequena a média duração que atuavam na internação dos hospitais.

Dados publicados em 2006, pelo Conselho Federal de Nutricionistas - CFN ${ }^{16}$ mostraram que a grande maioria dos nutricionistas é do sexo feminino. Segundo o CFN ${ }^{16}, 96,50 \%$ dos profissionais graduados eram mulheres. Alguns estudos vêm mostrando que essa característica é inerente às origens históricas desse profissional no Brasil e que tal peculiaridade continua reaparecendo nos dias atuais ${ }^{17}$.

Em relação ao tempo de formação, o predomínio de profissionais graduados há pelo menos 10 anos difere do encontrado pelo Conselho Federal de Nutricionistas ${ }^{16}$ que observou $49,90 \%$ dos nutricionistas com até cinco anos de graduação, seguido pelos que já haviam concluído o curso há mais de 10 anos (29,50\%).

Respostas divergentes às encontradas pelo $\mathrm{CFN}^{16}$ também foram encontradas em relação à instituição de graduação e a formação complementar. De acordo com o CFN, a maior parte dos profissionais graduou-se em instituições privadas $(56,30 \%)$ e, nem todos tinham formação complementar, tendo sido verificados os seguintes índices de realização: $47,40 \%$ cursos de especialização, $9,40 \%$ mestrado e $2,40 \%$ doutorado. No presente estudo observamos que a maioria se graduou em instituições públicas e todos deram continuidade aos estudos. Resultados mais próximos aos aqui encontrados foram obtidos no estudo de Rodrigues et $\mathrm{a} /{ }^{18} \mathrm{com}$ alunos egressos da Universidade $\mathrm{Fe}$ deral de Minas Gerais, onde verificou-se que $70 \%$ dos nutricionistas tinham concluído algum curso de pós-graduação, destes, $17 \%$ haviam feito mestrado e $4 \%$ doutorado.

Apesar do maior número de cursos de graduação em Nutrição no Estado do Rio de Janeiro ser oferecido por 20 instituições particulares, contra apenas 4 instituições públicas, no presente estudo observamos que a maioria dos profissionais atuantes nos hospitais estudados graduaram-se em Instituições de Ensino Superior - IES públi- 
cas. Segundo dados do último Relatório Síntese, divulgado pelo Instituto Nacional de Estudos em Pesquisas Educacionais Anísio Teixeira - INEP, gerado a partir do Exame Nacional de Desempenho dos Estudantes - ENADE $2013^{19}$, a média das notas das IES é, significativamente, maior nas públicas do que nas privadas, assim como as médias obtidas pelos estudantes de instituições públicas, havendo destaque para as médias obtidas principalmente na região sudeste do país. Tais fatores podem ser determinantes para a escolha da Universidade pública e também para um maior êxito, inclusive na aprovação em concursos públicos, dos profissionais nela formados, uma vez que os hospitais estudados pertenciam à esfera pública, onde normalmente, se faz necessário o ingresso por meio de concurso público.

Gambardella et $a^{20}$ observaram que apenas $37,10 \%$ dos nutricionistas continuaram estudando após o término da graduação, sendo que $29,20 \%$ na área de nutrição, fazendo cursos de especialização ou pós-graduação e 7,90\% prosseguiram adquirindo conhecimentos em outros assuntos, como cursos de língua estrangeira. Entre eles, $57,30 \%$ disseram não estar estudando e $5,60 \%$ não informaram. Como visto pelos autores, uma minoria dos entrevistados persistiram no aperfeiçoamento profissional, mostrando um resultado parecido com o que foi encontrado pelo $\mathrm{CFN}^{16}$.

Segundo as Diretrizes Curriculares Nacionais do Curso de Graduação em Nutrição ${ }^{21}$ a formação do nutricionista é generalista, ou seja, ele tem autonomia para atuar em diversas áreas. Então, dar continuidade aos estudos é imprescindível para a carreira deste profissional, pois ele necessita aprofundar os seus conhecimentos no ramo em que deseja atuar ${ }^{20}$. Outro aspecto importante é o aumento da renda, com a melhora do nível educacional ${ }^{17}$.

No estudo conduzido por Rodrigues et a/18 foi visto que a preferência de área para pós-graduação foi a nutrição clínica (67\%), seguida da alimentação coletiva (44\%). Esse resultado é semelhante ao encontrado em nossa pesquisa, onde a maior parte dos entrevistados (25\%) optou por ampliar seus estudos na área de nutrição do adulto que faz parte da nutrição clínica, predominantemente, atendido nos hospitais aqui estudados.

No trabalho de Gambardella et $a^{20}$ os temas mais procurados para a formação complemen- tar do nutricionista foram, em ordem decrescente: unidade de alimentação e nutrição - UAN, nutrição do atleta, nutrição clínica e marketing. Tais resultados não corroboram com os aqui encontrados, visto que alimentação coletiva foi a área menos escolhida. Para Souza et $a^{17}$, o gênero influência nas escolhas pelas áreas de atuação, observando-se maior interesse dos homens pelas áreas associadas a atributos da identidade masculina, como em academias ou clubes esportivos, enquanto as mulheres permanecem atuando principalmente na área da nutrição clínica ou nos restaurantes.

Independente da área escolhida para pós graduarem-se, a continuidade dos estudos foi apontada como ferramenta importante tanto para a atuação técnica (por exemplo, em cálculos de dieta) quanto para a melhora da humanização do profissional, mudando o olhar do nutricionista sobre o paciente, e também para a melhora do seu local de trabalho.

Conforme verificado, a maior parte dos nutricionistas atuava na área de internação e poucos atuavam no ambulatório. O mesmo foi encontrado pelo $\mathrm{CFN}^{16}$.

Para Zucch ${ }^{22}$ produzir saúde exige mão-de-obra intensiva, tendo em vista que os hospitais deveriam ser unidades para pacientes graves que requerem cuidados mais intensivos. Sendo assim, a melhoria da relação entre qualidade e quantidade dos profissionais é fundamental para uma melhor produtividade. $\mathrm{O}$ cálculo do dimensionamento de nutricionistas na área hospitalar ocorre com base no número de pacientes por nível de assistência, podendo este ser classificado em 3 níveis: primário, secundário e terciário ${ }^{29}$. Para determinar o nível de assistência é necessário conhecer a condição clínica do paciente, seu estado nutricional e a prescrição médica para definir a necessidade de atenção dietética especializada ${ }^{3}$. O cálculo do dimensionamento de nutricionistas, de acordo com a Resolução do CFN no $380 / 2005^{29}$, prevê que a nível secundário faz-se necessário 1 nutricionista a cada 30 pacientes, e a nível terciário 1 nutricionista a cada 15 pacientes. É importante lembrar que na prática, algumas situações clínicas podem atender a dois critérios $^{3}$. Dessa forma, conclui-se que a relação $n^{\circ}$ de nutricionista por leito, nos hospitais estudados, parece estar acima do preconizado, o que pode prejudicar a produtividade e a qualidade do atendimento prestado aos pacientes. 
Pedroso et $a^{24}$ destacam o quanto o cuidado nutricional assume fundamental importância dentro do processo de humanização no ambiente hospitalar. Na nutrição, o cuidado inclui a avaliação do estado nutricional do paciente; a identificação de metas terapêuticas; a escolha das intervenções a serem implementadas; a identificação das orientações necessárias; e a formulação de um plano de avaliação. E para a execução da intervenção faz-se necessário, entre diversas ações, a prescrição dietética que inclui, entre outros itens, o Valor Energético Total (VET) ${ }^{3}$.

Como visto acima, tanto a avaliação do estado nutricional do paciente quanto a avaliação de suas necessidades dietéticas estão incluídas no cuidado nutricional. Dentre os vários métodos objetivos existentes tem-se o antropométrico ${ }^{24}$. Segundo a ASBRAN ${ }^{3}$, no Brasil, apesar de considerar-se importante a triagem de risco nutricional em pacientes, não existe consenso sobre o melhor método a ser utilizado, sendo o IMC um indicador possível.

Para os profissionais participantes deste estudo, o IMC foi o método escolhido. Segundo Ricardo e Araújo ${ }^{11}$ e a ASBRAN ${ }^{3}$, o IMC deve ser utilizado com cautela, por não refletir a composição corporal e possuir limitações matemáticas intrínsecas. Entretanto, para Peixoto et a/25 o IMC tem ganho relevância nos estudos epidemiológicos por ser de fácil aplicação e utilizar medidas no seu cálculo que são de fácil obtenção, assim como por possibilitar classificar o estado antropométrico e o monitoramento do peso.

Como visto anteriormente, somente os profissionais de uma unidade hospitalar relataram ter acesso a leito balança e a bioimpedância. Sendo assim, o uso do IMC pelos nutricionistas, como mencionado, foi justificado por ser de fácil aplicação e não necessitar de equipamentos sofisticados, sendo necessário, apenas, a balança e o estadiômetro. A facilidade na aplicação deste indicador pode ser um fator determinante para sua escolha nos locais onde a relação número de profissionais por leito esteja prejudicada.

A associação de diferentes métodos objetivos (antropometria, composição corpórea, parâmetros bioquímicos e consumo alimentar) e subjetivos (exame físico e avaliação subjetiva global) pode ajudar a dirimir as possíveis interferências inerentes ao uso de apenas um método, podendo-se obter um diagnóstico nutricional mais adequado, principalmente, em nível individual e entre os pacientes mais graves ${ }^{7}$.

Outro fator que pode influenciar no diag- nóstico nutricional com base apenas no IMC é o uso de medidas autorelatadas. Apesar das medidas de peso e altura serem simples e de fácil obtenção, como visto na FIGURA 2, alguns nutricionistas $(28,12 \%)$ disseram não possuir estadiômetro em seu trabalho, podendo-se supor que tais profissionais utilizem a altura informada pelo paciente para o cálculo.

Enquanto Pedroso et a/ ${ }^{24}$ destacam o quanto é importante que as medidas antropométricas sejam coletadas pela equipe de saúde, pois quando referidas pelo paciente nem sempre são fidedignas, outros ${ }^{25,26}$ apontam que o IMC calculado a partir de medidas autoreferidas não difere, de forma significativa, do obtido a partir de medidas aferidas. Nesse contexto ressalta-se o quanto um diagnóstico adequado é ferramenta crucial para a tomada de decisão a respeito da prescrição dietoterápica.

Além do diagnóstico nutricional adequado, o estabelecimento das necessidades energéticas faz-se necessário ${ }^{3}$. Segundo a ASBRAN $^{3}$ condições clínicas alteram as necessidades de nutrientes para além do recomendado pela Ingestão Dietética de Referência. E para atender tais recomendações diferenciadas, vários consensos e diretrizes nacionais e internacionais têm sido publicados, por diferentes instituições. No que tange ao GET, muitas dessas recomendações diferenciadas são feitas com base na distribuição de Kcal por Kg de peso, conforme determinado pela The European Society for Clinical Nutritionand Metabolism (ES$\mathrm{PEN}^{15}$ ), pela Sociedade Brasileira de Alimentação e Nutrição (SBAN ${ }^{13}$ ), pela Sociedade Brasileira de Diabetes $\left(\mathrm{SBD}^{14}\right)$, entre outras.

Popularmente conhecida como Fórmula ou Regra de Bolso, o método $\mathrm{Kcal} / \mathrm{Kg}$ de peso tem sido divulgado como uma opção de cálculo da necessidade energética, em substituição aos métodos como os de calorimetria indireta, conforme descrito pela SBAN ${ }^{13}$. No presente estudo, esse foi o método escolhido pelos profissionais, tanto na internação quanto no ambulatório, independentemente, do conhecimento de outros métodos de cálculo.

Resultados semelhantes aos nossos foram encontrados por Cunha et $a^{27}$ ao avaliarem 112 questionários respondidos por intensivistas, onde $60 \%$ utilizavam a regra de bolso $25-30 \mathrm{kcal} / \mathrm{kg}$ de peso e apenas $35 \%$ utilizavam a equação de Harris e Benedict.

Apesar do frequente uso do método Kcal/ Kg de peso e a indicação na prática clínica, Ricardo e Araújo ${ }^{11}$ destacam que existem ainda inúmeras 
restrições teóricas ao uso e às faixas de normalidade preconizadas.

Em 2011, a FAO/OMS ${ }^{28}$ revisou os métodos para o cálculo das necessidades energéticas humanas em diferentes fases da vida. No grupo dos adultos, por exemplo, apesar de verificarem grande variação de necessidades energéticas verificou-se que a taxa metabólica basal era relativamente constante entre classes de indivíduos de uma determinada idade e sexo, o que levou alguns autores a concluírem que o peso corporal e a atividade física são as principais causas para a diversidade nas necessidades energéticas. No citado documento também foram descritas faixas de recomendação de energia por $\mathrm{Kcal} / \mathrm{Kg}$ de peso para homens e mulheres, em diferentes momentos da vida e ressaltou-se que esse intervalo pode ser alterado dependendo do nível de atividade física. Tais determinações foram baseadas em necessidades energéticas de grupos de indivíduos semelhantes e saudáveis, sendo assim pode-se inferir que o emprego desses resultados com intenções terapêuticas ou outros, deve levar em consideração a possibilidade de erros de análise e procedimentos inadequados.

Como afirmado, os motivos apontados para a escolha do método $\mathrm{Kcal} / \mathrm{Kg}$ de peso são a praticidade e a rapidez no cálculo. Poucos profissionais reconheceram limitações no uso do referido método, o que é preocupante, uma vez que são comuns estudos que apontam erros intrínsecos ao uso de diferentes métodos de cálculo de energia, derivados de equações preditivas ${ }^{10,29}$. Da mesma forma, apesar de poucos estudos ${ }^{5,11}$ referirem limitações especificamente quanto ao uso do método $\mathrm{Kcal} / \mathrm{Kg}$ de peso, elas existem.

Dessa maneira, a publicação de recomendações específicas para determinadas patologias é imprescindível para dirimir erros, devendo-se buscar documentos que orientem faixas de $\mathrm{Kcal} /$ $\mathrm{Kg}$ de peso que sejam constantemente revisados por renomadas instituições. Além disso, outros pontos nevrálgicos são o diagnóstico nutricional adequado que acaba por influenciar na escolha do peso a ser utilizado para cálculo e, também, a escolha do valor que será multiplicado a esse peso, visto que são recomendadas faixas de $\mathrm{Kcal} / \mathrm{Kg}$.

Segundo Farcy et $a^{\beta 0}$, apesar da calorimetria indireta ser o padrão ouro para o estabelecimento das necessidades calóricas em pacientes gravemente enfermos, o uso de métodos como o $\mathrm{de} \mathrm{Kcal/Kg}$ de peso deve ser aplicado por ser uma alternativa mais simples, nas instituições onde a medida por calorimetria indireta não seja viável, considerando que estudos tem apontado limitações no uso de equações preditivas.

Para Segadilha et $a^{5}$ a equação de Harris e Benedict corrigida apresentou limites de concordância mais próximos das leituras de gasto energético em repouso, medido por calorimetria indireta, ao contrário da recomendação de 20 a $25 \mathrm{Kcal} / \mathrm{Kg}$ de peso atual ${ }^{15}$ que apresentou os piores resultados. Segundo os autores, apesar do método Kcal/ $\mathrm{Kg}$ de peso ser uma maneira simples de prever o gasto energético em repouso, a recomendação do ESPEN ${ }^{15}$ pode promover a hiperalimentação na prática clínica em idosos admitidos na UTI, provavelmente, por só levar em consideração a variável peso para cálculo.

O conhecimento de possíveis erros intrínsecos ao método escolhido, assim como o diagnóstico e o acompanhamento do estado nutricional dos pacientes são fundamentais para o estabelecimento de prescrições dietéticas adequadas, tornando possível a correção de prescrições aquém ou além do necessário, evitando-se efeitos deletérios aos pacientes.

Embora os resultados sejam de grande interesse para o entendimento da prática clínica de nutricionistas no que tange, principalmente, a avaliação do estado nutricional e ao cálculo de necessidade energética, algumas limitações podem ser atribuídas ao estudo. Em primeiro lugar, tivemos dificuldade no contato com os órgãos superiores responsáveis por autorizar a participação das unidades hospitalares. E, por fim, tivemos uma adesão limitada dos nutricionistas nas unidades participantes, o que pode ter influenciando a análise. Contudo, os resultados indicam o quanto estudos deste tipo podem contribuir para reflexões sobre a prática dos profissionais e os fatores que a influenciam.

\section{Conclusão}

Apesar do conhecimento de diferentes métodos de avaliação nutricional e do cálculo da necessidade energética, existiu preferência pelo IMC e pelo método $\mathrm{Kcal} / \mathrm{Kg}$ de peso, respectivamente, independentemente, do local de atuação ou especificidade no tratamento.

A facilidade e a praticidade na aplicação, assim como o pouco reconhecimento de suas limitações foram preponderantes para tais escoIhas. Entre outros motivos destacam-se a falta de equipamentos e o número de profissionais por enfermaria. 


\section{REFERÊNCIAS}

1. Bottoni A, Hassan DZ, Nacarato A, Garnes SA, Bottoni A. Porque se preocupar com a desnutrição hospitalar? J Health Scilnst. 2014; 32(3): 314-7.

2. Waitzberg DL, Caiaffa WT, Correia MITD. Hospital Malnutrition: The Brazilian National Survey (IBRANUTRI): A Study of 4000 Patients Nutrition, 2001; 17(7/8).

3. ASBRAN - Associação Brasileira de Nutrição. Manual Orientativo: Sistematização do Cuidado de Nutrição. São Paulo: Associação Brasileira de Nutrição; 2014; 66p.

4. Weekes CE. Controversies in the determination of energy requirements. Proceedings of the Nutrition Society. 2007; 66: 367-77.

5. Segadilha NLA, Rocha EEM, Tanaka LMS, Gomes KLP, Espinoza REA, Peres WAF. Energy Expenditure in Critically III Elderly Patients: Indirect Calorimetry vs Predictive Equations. Journal of Parenteral and Enteral Nutrition. 2016; 20(10): 1 - 9

6. Macdonald A, Hildebrandt L. Comparison of formulaic equations to determine energy expenditure in the critically III patient. Nutrition, United States. 2003; 19(3): 233-239.

7. Pereira TCF. Diferentes Métodos de Avaliação do Metabolismo de Repouso em Mulheres Obesas Antes e Após Cirurgia Bariátrica. Faculdade de ciências da nutrição e alimentação, Porto; 2015

8. Volp ACP, Oliveira FCE, Alves DM, Esteves EA, Bressan J. Energy expenditure: components and evaluation methods. Nutrición Hospitalaria. 2011; 26:430-40.

9. Nonino CB. Calorimetria indireta $X$ Harris Benedict: determinação, validação e comparação para cálculo da taxa metabólica de repouso em obesos grau III. Ribeirão Preto, 2002. 111 f. Dissertação (Mestrado em Clínica Médica) - Faculdade de Medicina de Ribeirão Preto.

10. Wahrlich $\vee$, Anjos LA. Validação de equações de predição da taxa metabólica basal em mulheres residentes em Porto Alegre, RS, Brasil. Rev Saúde Pública. 2001; 35:39-45.

11. Ricardo DR, Araújo CGS. Índice de Massa Corporal: Um Questionamento Científico Baseado em Evidências. Arq. Bras. Cardiol. 2002; 79(1): 61-9.

12. Sanches ACS. Avaliação do gasto energético de repouso em pacientes com sepse associada ou não à lesão renal aguda. Universidade Estadual Paulista "Júlio de Mesquita Filho", Faculdade de Medicina, Botucatu; 2016.

13. SBAN - Sociedade Brasileira de Alimentação e Nutrição. Terapia Nutricional em Pacientes Críticos. Disponível em: http://www.sban.org.br/por_dentro/informativos/123/ terapia-nutricional-em-pacientes-criticos.

14. SBD - Sociedade Brasileira de Diabetes. Diretrizes SBD (2015-2016). São Paulo: A.C. Farmacêutica, 2016.

15. ESPEN - The European Society for Clinical Nutrition and Metabolism. ESPEN Guidelines. Disponível em: http:// www.espen.org/education/espen-guidelines.

16. CONSELHO FEDERAL DE NUTRICIONISTAS - CFN. Perfil da atuação profissional do nutricionista no Brasil. Brasília: CFN, 2006; p. 88. Disponível em: http://www.cfn. org.br/novosite/pdf/pesquisa.pdf.

17. Souza LKCS, Campos FM, Kraemer FB, Machado PA, Carvalho MCVS, Prado SD. Gênero e formação profissional: considerações acerca do papel feminino na construção da carreira de nutricionista. Demetra: Alimentação, Nutrição \& Saúde. 2016; 11(3): 773-788.
18. Rodrigues KM, Peres F, Waissmann W. Condições de trabalho e perfil profissional dos nutricionistas egressos da Universidade Federal de Ouro Preto, Minas Gerais, entre 1994 e 2001. Ciência \& Saúde Coletiva. 2007; 12 (4):1021- 1031

19. BRASIL. Ministério da Educação. Instituto Nacional de Estudos e Pesquisas Educacionais Anísio Teixeira - INEP. Relatório de Área - Exame Nacional de Desempenho dos Estudantes - ENADE 2013.

20. Gambardella AMD, Ferreira CF, Frutuoso MFP. Situação profissional de egressos de um curso de nutrição. Rev. Nutr. Campinas. 2000; 13(1): 37 -40.

21. BRASIL. Resolução CNE/CES n 5 , de 7 nov. 2001. Institu Diretrizes Curriculares Nacionais do Curso de Graduação em Nutrição. Disponível em: http://portal.mec.gov.br/cne/ arquivos/pdf/CES05.pdf.

22. Zucch P. Funcionários por leito: estudo em alguns hospitais públicos e privados. RAP. 1998; 32(3):65-76.

23. Conselho Federal de Nutricionistas. Resolução CFN no 380/2005. Brasília: CFN; 2005 [acesso em 2017 jun 17]. Disponivel em: http://www.cfn.org.br/novosite/pdf/ res/2005/res380.pdf

24. Pedroso CGT, Sousa AA, Salles RK. Cuidado nutricional hospitalar: percepção de nutricionistas para atendimento humanizado. Ciência \& Saúde Coletiva. 2011; 16(Supl. 1):1155-1162.

25. Peixoto MRG, Benício MHD, Jardim, PCBV. Validade do peso e da altura auto-referidos: o estudo de Goiânia. Rev Saúde Pública. 2006; 40(6):1065-72.

26. Oliveira LPM, Queiroz VAO, Silva MCM, Pitangueira JCD Costa PRF, Demétrio F, Anjos MCG, Assis AMO. Índice de massa corporal obtido por medidas autorreferidas para a classificação do estado antropométrico de adultos: estudo de validação com residentes no município de Salvador estado da Bahia, Brasil. Epidemiol. Serv. Saúde, Brasília. 2012; 21(2): 325-332.

27. Cunha HFR, Salluh JIF, França MF. Atitudes e percepções em terapia nutricional entre médicos intensivistas: um inquérito via internet. Rev. Bras. Ter Intensiva, 2010 22(1): 53-63.

28. FAO/WHO/UNU. Report of Joint Expert.Consultation. Food and Nutrition Technical Report Series 1. Human Energy Requirements. Rome, 2001.

29. Cruz CM, Silva AF, Anjos LA. A taxa metabólica basal é superestimada pelas equações preditivas em universitárias do Rio de Janeiro, Brasil. Arch Latinoam Nutr. 1999; 49: 232-7.

30. Farcy DA, Chiu WC, Flaxman A, Marshall JP. Cuidados intensivos na medicina de emergência. AMGH editora, $1^{\circ} \mathrm{ed}$.; 2013.

\section{CORRESPONDÊNCIA}

Camila Ramos Berniz

Av. Pasteur, 296 - Urca

CEP: 22290-902 - RIO DE JANEIRO - RJ

E-mail: cep.unirio09@gmail.com 